\title{
The Phase Transition of Higher Dimensional Charged Black Holes
}

\author{
Xiongying Guo, ${ }^{1,2}$ Huaifan Li, ${ }^{1,2}$ Lichun Zhang, ${ }^{1,2}$ and Ren Zhao ${ }^{1,2}$ \\ ${ }^{1}$ Institute of Theoretical Physics, Shanxi Datong University, Datong 037009, China \\ ${ }^{2}$ Department of Physics, Shanxi Datong University, Datong 037009, China \\ Correspondence should be addressed to Huaifan Li; huaifan.li@stu.xjtu.edu.cn
}

Received 17 February 2016; Accepted 24 April 2016

Academic Editor: George Siopsis

Copyright (c) 2016 Xiongying Guo et al. This is an open access article distributed under the Creative Commons Attribution License, which permits unrestricted use, distribution, and reproduction in any medium, provided the original work is properly cited. The publication of this article was funded by $\mathrm{SCOAP}^{3}$.

\begin{abstract}
We have studied phase transitions of higher dimensional charge black hole with spherical symmetry. We calculated the local energy and local temperature and find that these state parameters satisfy the first law of thermodynamics. We analyze the critical behavior of black hole thermodynamic system by taking state parameters $(Q, \Phi)$ of black hole thermodynamic system, in accordance with considering the state parameters $(P, V)$ of van der Waals system, respectively. We obtain the critical point of black hole thermodynamic system and find that the critical point is independent of the dual independent variables we selected. This result for asymptotically flat space is consistent with that for AdS spacetime and is intrinsic property of black hole thermodynamic system.
\end{abstract}

\section{Introduction}

In recent years, the study of critical behavior of black holes has still received a lot of attention. Particularly, the idea of including the variation of the cosmological constant $\Lambda$ in the first law of black hole thermodynamics has attained increasing attention. Matching the thermodynamic quantities with the ones in usual thermodynamic system, the critical behavior of black holes can be investigated and the phase diagram like the van der Waals vapor-liquid system, critical exponents, and Clapeyron equations can be obtained [1-14]. For the de Sitter spacetime, the thermodynamic properties and phase transition of black hole with the method of the equivalence quantities have been investigated, and it is shown that the result is similar to AdS black hole [15-20]. This helps to further understand black hole entropy, temperature, heat capacity, and so on, and it is also very important to improve the self-consistent geometric theory of black hole thermodynamics.

As is well known, there are Hawking radiations for black holes in asymptotically flat spacetime and nonasymptotically flat ones. Because the heat capacity of black hole in asymptotically flat spacetime is negative, this black hole in asymptotically flat space is thermodynamically unstable. For studying the thermodynamics properties and phase transition of black hole, we should firstly verify that the black hole is thermodynamically stable.

In order to restore thermodynamic stability so that equilibrium thermodynamics and the phase structure can be studied, we must consider the whole systems that include not only the black hole under consideration but also its environment [21, 22], as self-gravitating systems are spatially inhomogeneous, which is different from the usual thermodynamic system. Any specification of such system requires not only thermodynamic quantities of interest but also the place at which they take the specified values. In this paper, we have studied the phase transition of higher dimensional charged black hole with spherical symmetry. To solve this problem we can place the black hole inside a finite concentric spherical cavity, whose radii are fixed and larger than the one of black hole. The temperature is fixed on the surface of the cavity, which could be physically realized by placing a heat bath around the cavity. We will keep the charge inside the cavity also fixed. This will define a canonical ensemble [23-28]. 
We will study the phase structure and the thermodynamic properties of the various dimensional charged black hole in this ensemble.

References [29-35] have investigated the critical behavior of various black holes in AdS spacetime with Ehrenfest scheme. They found that the phase transition of black hole is the continuous one in AdS spacetime, and the thermodynamic quantities of the critical point satisfy the Ehrenfest equation. References [36-38] have obtained the same conclusion by studying the thermodynamics and state space geometry of black hole in AdS space. Moreover, it is interesting to study the thermodynamics behavior of the ensemble, which we construct for asymptotical flat space, and identify whether the ensemble meets the Ehrenfest equation. We obtain the phase diagram for the thermodynamic quantities $(Q, \Phi)$ at the critical point and the result similar to that in AdS black hole. The thermodynamic quantities satisfy the Ehrenfest equation, so the corresponding phase transition is a continuous phase transition.

The paper is arranged as follows: In Section 2 we first review the higher dimensional charged black hole in asymptotically flat space and give the quasilocal thermodynamic quantities of the canonical ensemble. In Section 3 the critical behavior in different dimensional charged black hole is investigated. Finally, the paper ends with a brief conclusion (we use the units $G_{d}=\hbar=k_{B}=c=1$ ).

\section{The Thermodynamical Quantity of the Charged Black Hole with the Ensemble Theory in Higher Dimension}

The solution for charged black hole in spacetime dimensions with $d>3$ reads

$$
d s^{2}=-V d t^{2}+\frac{d r^{2}}{V}+r^{2} d \Omega_{d-2}^{2},
$$

where the $V(r)$ functions entering the metric are given by

$$
V(r)=1-\frac{m}{r^{d-3}}+\frac{q^{2}}{r^{2(d-3)}} \text {. }
$$

Here the parameter $m$ is related to the ADM mass $(M)$ of black holes:

$$
M=\frac{(d-2) \omega_{d-2}}{16 \pi} m ; \quad \omega_{d-2}=\frac{2 \pi^{(d-1) / 2}}{\Gamma((d-1) / 2)},
$$

where $\omega_{d-2}$ is the volume of unit $(d-2)$ sphere. The parameter $q$ is related to the electric charged $Q$ as

$$
Q=\frac{\sqrt{2(d-2)(d-3)}}{8 \pi} \omega_{d-2} q .
$$

The entropy of the system is given by

$$
S=\frac{\omega_{d-2}}{4} r_{+}^{d-2}
$$

where $r_{+}$is the radius of the outer event horizon defined by the condition $V\left(r_{+}\right)=0$. Let us first consider the cavity as a boundary with a radius $r_{B}$ to study quasilocal thermodynamics along the line of the procedure in [22]. Then, the local temperature measured at the boundary is given by [22-28]

$$
T_{\mathrm{loc}}=\frac{T}{\sqrt{V\left(r_{B}\right)}}=\frac{(d-3)\left(r_{+}^{2(d-3)}-q^{2}\right)}{4 \pi \sqrt{V\left(r_{B}\right)} r_{+}^{2 d-5}} .
$$

For fixed charge $Q$, the entropy calculated from the first law of thermodynamics is

$$
S=\int \frac{d M}{T} .
$$

Applying the first law of thermodynamics, the total thermodynamic internal energy within the boundary $r_{B}$ is obtained as

$$
\begin{aligned}
E_{\mathrm{loc}} & =\int_{M_{0}}^{M} T_{\mathrm{loc}} d S=\int_{M_{0}}^{M} \frac{T_{\mathrm{loc}}}{T} d M \\
& =\frac{(d-2) \omega_{d-2}}{8 \pi} r_{B}^{d-3}\left(\sqrt{V\left(B_{0}\right)}-\sqrt{V\left(r_{B}\right)}\right) .
\end{aligned}
$$

Taking $V\left(B_{0}\right)=1$ at the boundary of cavity [26], we can obtain

$$
E_{\mathrm{loc}}=\frac{(d-2) \omega_{d-2}}{8 \pi} r_{B}^{d-3}\left(1-\sqrt{V\left(r_{B}\right)}\right),
$$

where

$$
V\left(r_{B}\right)=\left(1-\frac{r_{+}^{d-3}}{r_{B}^{d-3}}\right)\left(1-\frac{q^{2}}{r_{+}^{d-3} r_{B}^{d-3}}\right) .
$$

So we can define the reduction quantities for corresponding

$$
\begin{aligned}
& x=\frac{r_{+}^{d-3}}{r_{B}^{d-3}}, \\
& \tilde{q}=\frac{q}{r_{B}^{d-3}}, \\
& b_{q}(x)=\frac{(d-2) \beta_{B}}{8 \pi r_{B}}, \\
& h=\frac{(d-2) \omega_{d-2}}{8 \pi} r_{B}^{d-3}\left(1-\sqrt{(1-x)\left(1-\frac{\tilde{q}^{2}}{x}\right)}\right), \\
& S=\frac{\omega_{d-2}}{4} r_{B}^{d-2} x^{(d-2) /(d-3)} .
\end{aligned}
$$

For $r_{+}>q$ and $r_{B}>r_{+}$, we get $q<x<1$. The states functions in the cavity satisfy the first law of thermodynamic system $[22,39]$ :

$$
d E_{\mathrm{loc}}=T_{\mathrm{loc}} d S+\Phi d Q+\sigma d A
$$

where

$$
\begin{aligned}
& S=\frac{\omega_{d-2}}{4} r_{+}^{d-2}, \\
& A=\omega_{d-2} r_{B}^{d-2} .
\end{aligned}
$$




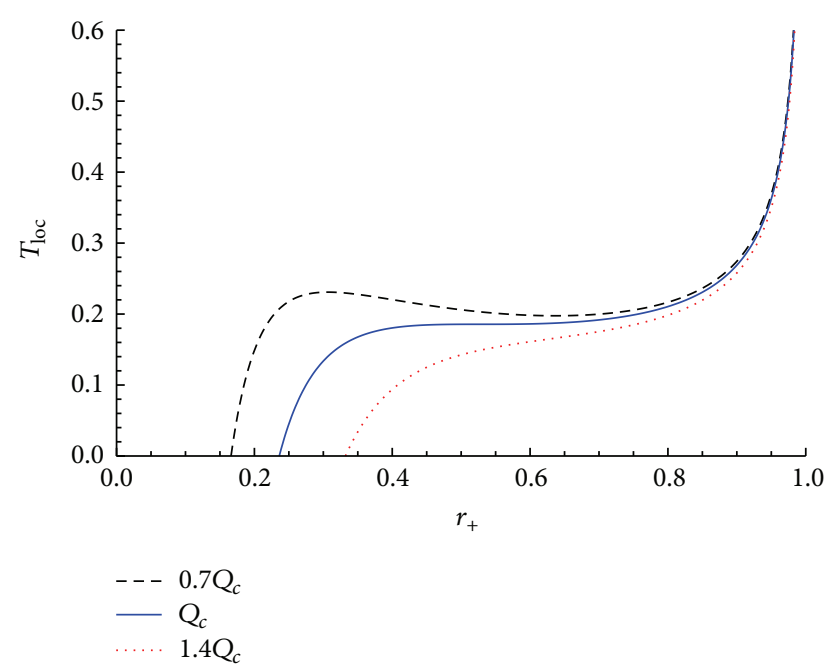

(a) $d=4$

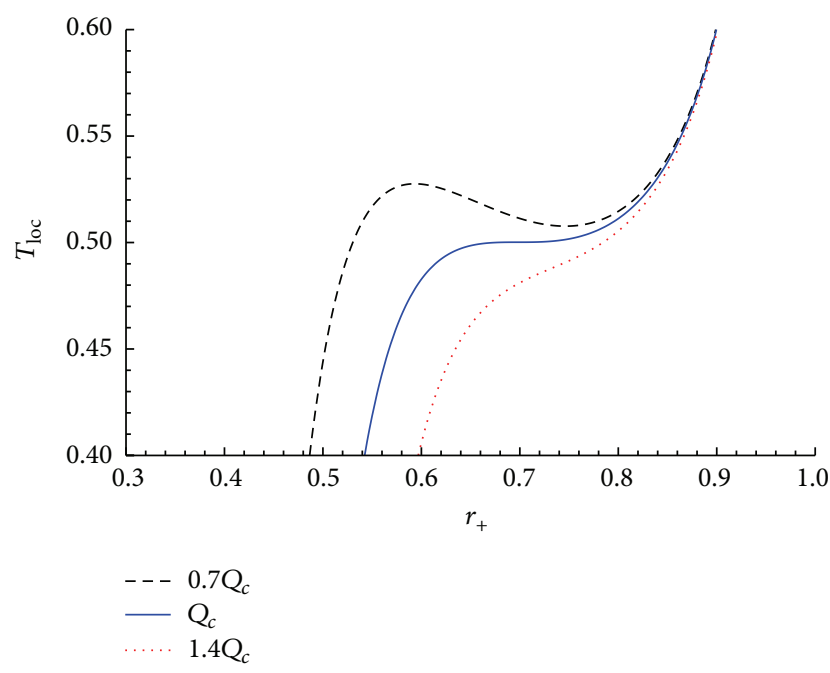

(b) $d=7$

FIGURE 1: The $T_{\text {loc }}-r_{+}$curves for $d=4,7$, respectively. From top to bottom the curves correspond to the charges $0.7 Q_{c}, Q_{c}$, and $1.4 Q_{c}$.

From (9) and (12), we can obtain

$$
\begin{aligned}
& \left(\frac{\partial E_{\mathrm{loc}}}{\partial S}\right)_{\mathrm{Q}, A}=T_{\mathrm{loc}} \\
& =\frac{(d-3)\left(1-q^{2} / r_{+}^{2(d-3)}\right)}{4 \pi r_{+}\left(1-r_{+}^{d-3} / r_{B}^{d-3}\right)^{1 / 2}\left(1-q^{2} / r_{+}^{d-3} r_{B}^{d-3}\right)^{1 / 2}}, \\
& \left(\frac{\partial E_{\mathrm{loc}}}{\partial Q}\right)_{S, A}=\Phi \\
& =\frac{(d-2) q\left(1-r_{+}^{d-3} / r_{B}^{d-3}\right)}{\sqrt{2(d-2)(d-3)} r_{+}^{d-3}\left(1-r_{+}^{d-3} / r_{B}^{d-3}\right)^{1 / 2}\left(1-q^{2} / r_{+}^{d-3} r_{B}^{d-3}\right)^{1 / 2}}, \\
& \left(\frac{\partial E_{\mathrm{loc}}}{\partial A}\right)_{r_{+}, \mathrm{Q}}=\sigma \\
& =\frac{(d-3)}{8 \pi r_{B}}\left[1-\frac{1-r_{+}^{d-3} / 2 r_{B}^{d-3}-q^{2} / 2 r_{+}^{d-3} r_{B}^{d-3}}{\left(1-r_{+}^{d-3} / r_{B}^{d-3}\right)^{1 / 2}\left(1-q^{2} / r_{+}^{d-3} r_{B}^{d-3}\right)^{1 / 2}}\right] .
\end{aligned}
$$

\section{The Critical Effect in Charged Black Hole in Cavity}

For the thermodynamic ensemble, we take the state parameters $T_{\text {loc }}, S$ as the independent variables to study the critical behaviors of thermodynamic ensemble. When these state parameters $Q$ and $r_{B}$ are invariable quantities, the critical values of black hole radii $r_{B}$, black hole event horizon $r_{+}$, and the black hole temperature are decided by

$$
\begin{gathered}
\left(\frac{\partial T_{\mathrm{loc}}}{\partial r_{+}}\right)_{r_{B}, Q}=0, \\
\left(\frac{\partial^{2} T_{\mathrm{loc}}}{\partial r_{+}^{2}}\right)_{r_{B}, Q}=0 .
\end{gathered}
$$

TABLE 1: Numerical solutions for $r_{+}^{c}, Q^{c}, T_{\text {loc }}^{c}$, and $\Phi^{c}$ for given values of $d=4,5,6,7$, respectively.

\begin{tabular}{ccccc}
\hline$d$ & $r_{+}^{c}$ & $Q^{c}$ & $T_{\text {loc }}^{c}$ & $\Phi^{c}$ \\
\hline 4 & 0.527864 & 0.236068 & 0.185589 & 0.324920 \\
5 & 0.610537 & 0.320704 & 0.301913 & 0.221054 \\
6 & 0.661764 & 0.378834 & 0.404099 & 0.177014 \\
7 & 0.698081 & 0.404347 & 0.500148 & 0.151505 \\
\hline
\end{tabular}

We can calculate the position of the critical points in different dimensional spacetime. The results are shown in Table 1 (we take $r_{B}=1$ ).

Table 1 shows that the critical values of $r_{+}^{c}, Q^{c}$, and $T_{\text {loc }}^{c}$ increase as the spacetime dimension $d$ increases, and $\Phi^{c}$ decreases as the spacetime dimension $d$ increases. Figure 1 shows $T_{\text {loc }}$ curves with black hole radii for different $d$. we can find that there is a phase transition near critical point with the charge $Q \leq Q_{c}$. The critical temperature $T_{\text {loc }}$ increases as the spacetime dimension $d$ increases. From the heat capacity of system

$$
C_{\mathrm{Q}}=T_{\mathrm{loc}}\left(\frac{\partial S}{\partial T_{\mathrm{loc}}}\right)
$$

we can find that the instability state will appear with the charge of spacetime less than the critical charge. By using Maxwell's equal area law we discover the possible two-phase coexistence curves in the process of phase transition $[39,40]$.

Next, let us calculate the free energy of the black hole in order to study phase transition between the black holes and the hot flat space $[24,28]$

$$
F=E_{\mathrm{loc}}-T_{\mathrm{loc}} S .
$$




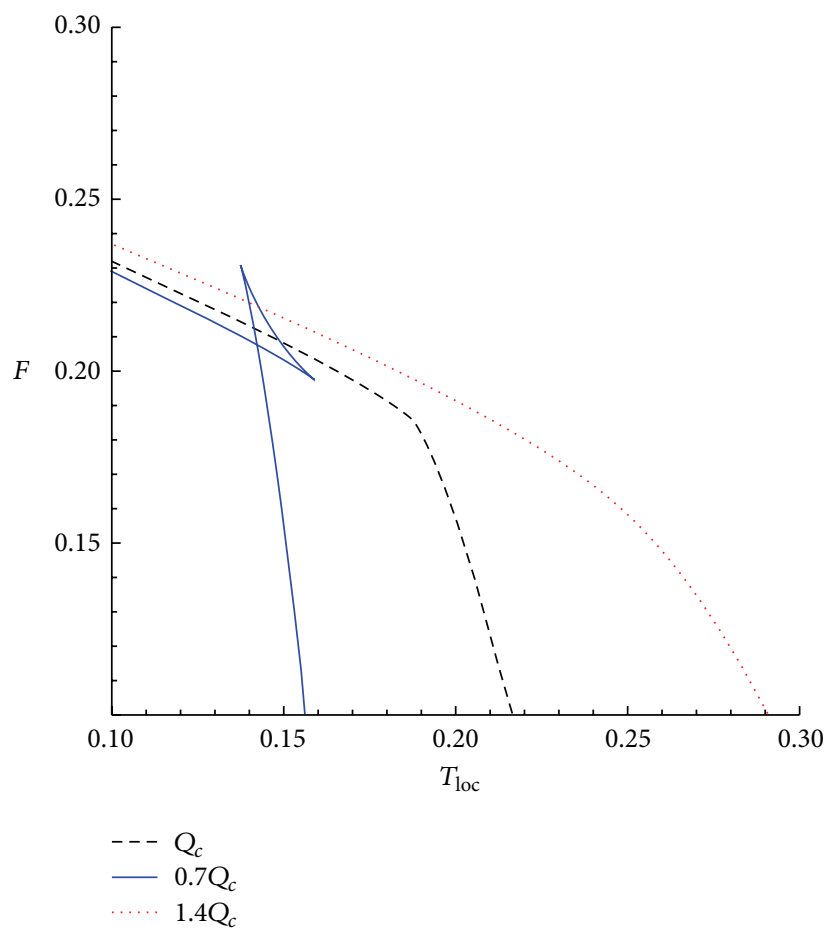

(a) $d=4$

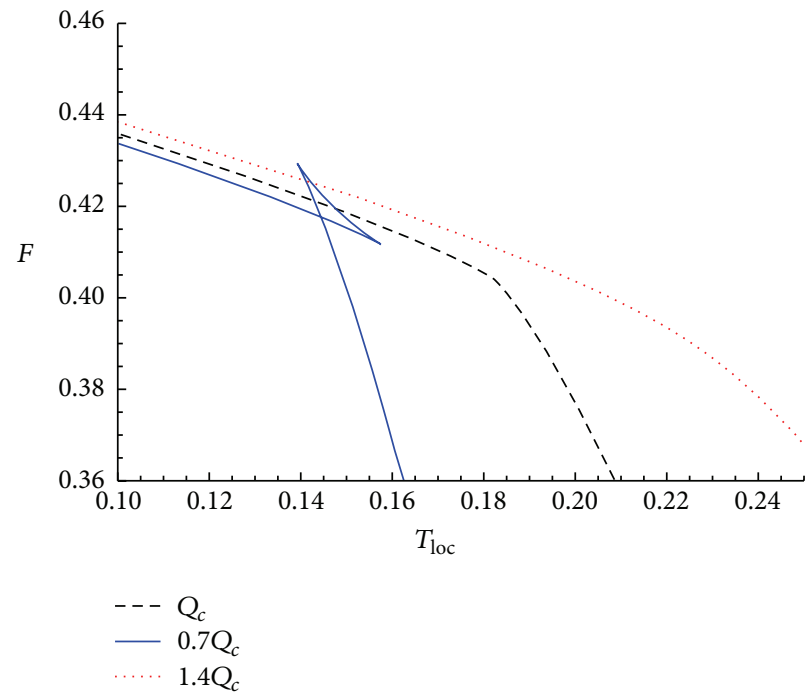

(b) $d=6$

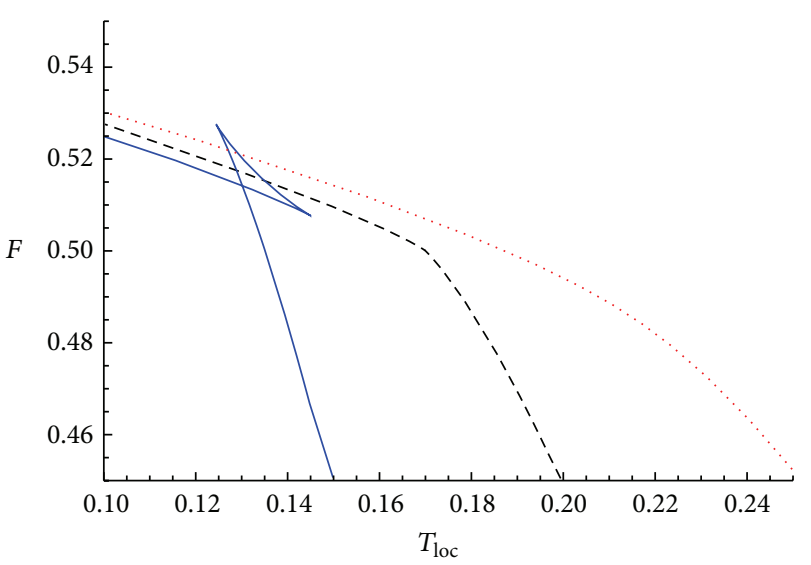

-- $Q_{c}$

$0.7 Q_{c}$

$1.4 Q_{c}$

(c) $d=7$

Figure 2: The $F-T_{\text {loc }}$ curves for $d=4,6,7$, respectively. From top to bottom the curves correspond to the effective temperatures $0.7 Q^{c}, Q^{c}$, and $1.4 Q^{c}$.

We can plot the relations curve for the free energy $F$ and the local temperature $T_{\text {loc }}$, with $r_{B}=1$, and $Q$ takes values near critical charge. Figure 2 shows that the system is the two-phase coexistence state, when the charge of black hole is smaller than the critical charge $Q_{c}$. This result is consistent with Figure 1. From Table 1, it is found that the critical temperature and critical charge increase as the spacetime dimension increases. This result is consistent with the conclusion in [3] for higher dimensional AdS black hole.
Further, We can plot the curve for the free energy $F$ and the local temperature $Q$, with $r_{B}=1$, and $T_{\text {loc }}$ take values near critical temperature in Figure 3. From Figure 3, we can see that there is a phase transition at the quasilocal temperature less than the critical quasilocal temperature in different dimensions. The results are consistent with the profile of $F-T_{\text {loc }}$ in Figure 2.

From (12), we know that we can select the independent variables $(Q, \Phi)$ or $(\sigma, A)$ for the black hole thermodynamic 


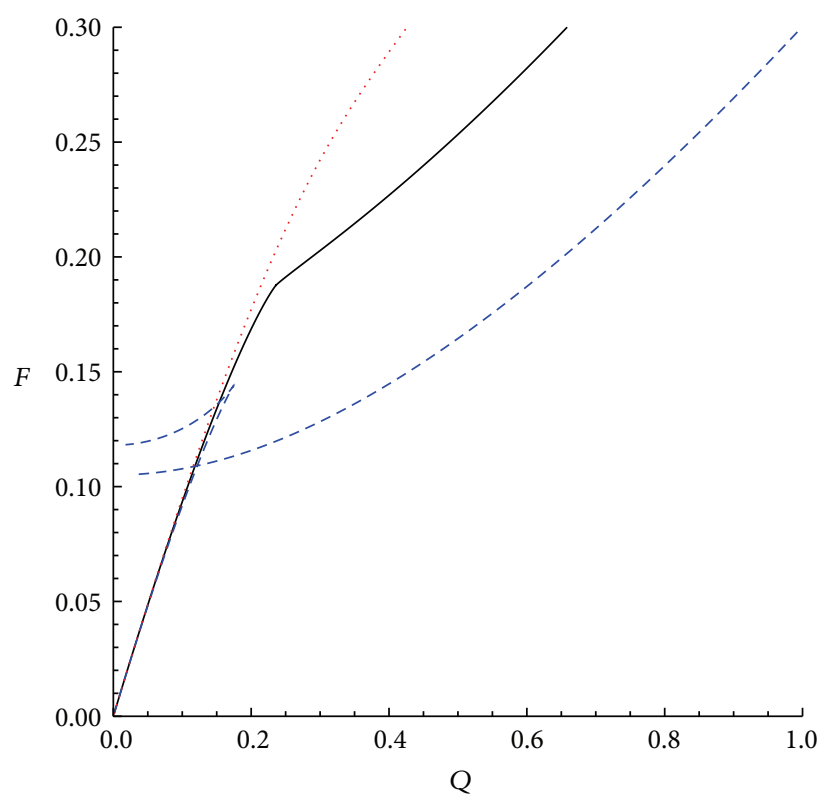

(a) $d=4$

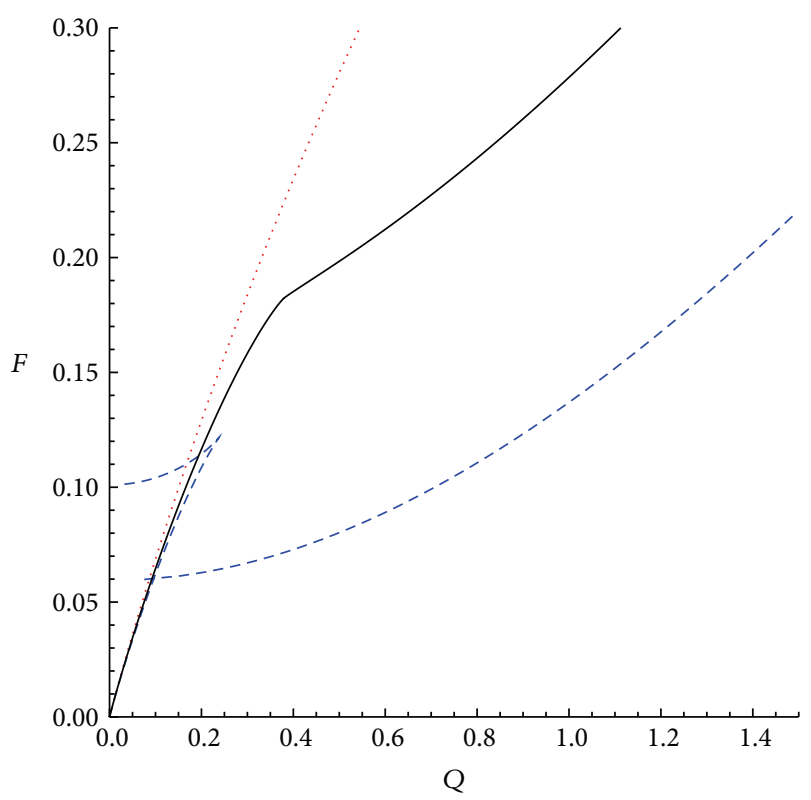

(b) $d=6$

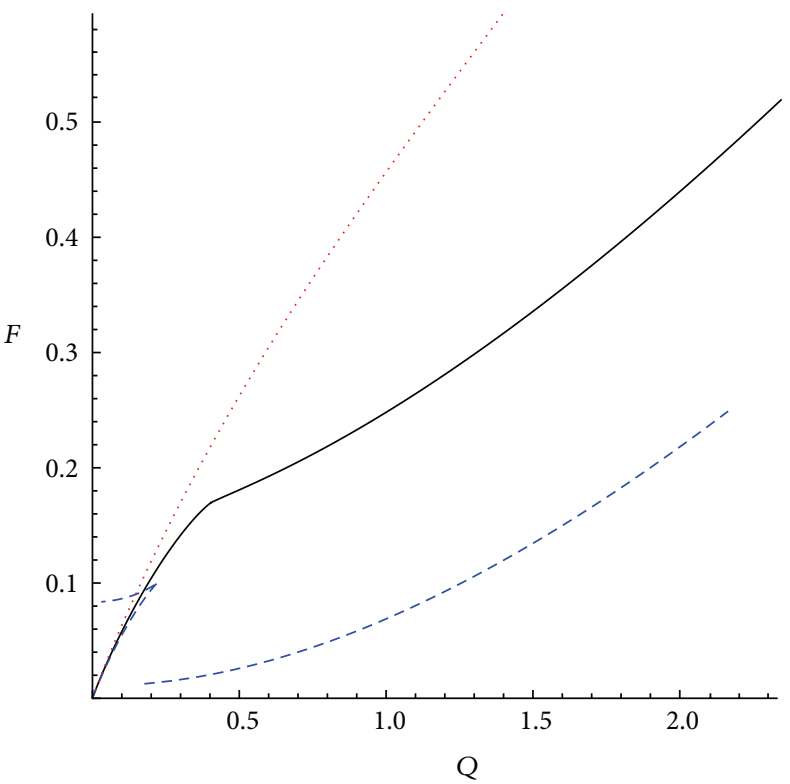

(c) $d=7$

Figure 3: The F-Q curves for $d=4,6,7$, respectively. From top to bottom the curves correspond to the effective temperatures $0.7 T_{\text {loc }}, T_{\text {loc }}$, and $1.4 T_{\mathrm{loc}}$.

ensemble. When taking $r_{B}$ and $T_{\text {loc }}$ as constants, we select the dual independent variables $(\Phi, Q)$, and the critical position is given by the following conditions:

$$
\begin{aligned}
& \left(\frac{\partial Q}{\partial \Phi}\right)_{T_{\mathrm{loc}}, r_{B}} \\
& =\frac{\left(\partial T_{\mathrm{loc}} / \partial r_{+}\right)_{\mathrm{Q}}}{(\partial \Phi / \partial Q)_{r_{+}}\left(\partial T_{\mathrm{loc}} / \partial r_{+}\right)_{\mathrm{Q}}-\left(\partial T_{\mathrm{loc}} / \partial Q\right)_{r_{+}}\left(\partial \Phi / \partial r_{+}\right)_{\mathrm{Q}}}
\end{aligned}
$$

$$
\begin{aligned}
& =\frac{\left(\partial T_{\mathrm{loc}} / \partial r_{+}\right)_{\mathrm{Q}}}{\partial\left(\Phi, T_{\mathrm{loc}}\right) / \partial\left(Q, r_{+}\right)}=f\left(r_{+}, Q\right)=0 \\
& \left(\frac{\partial^{2} \Phi}{\partial Q^{2}}\right)_{T_{\mathrm{loc},} r_{B}}=\left(\frac{\partial f\left(r_{+}, Q\right)}{\partial Q}\right)_{T_{\mathrm{loc},}, r_{B}}=0 .
\end{aligned}
$$

We can plot the curve for the free energy $\Phi$ and the local temperature $Q$ in Figure 4, with $r_{B}=1$, and $T_{\text {loc }}$ take values near critical temperature. 


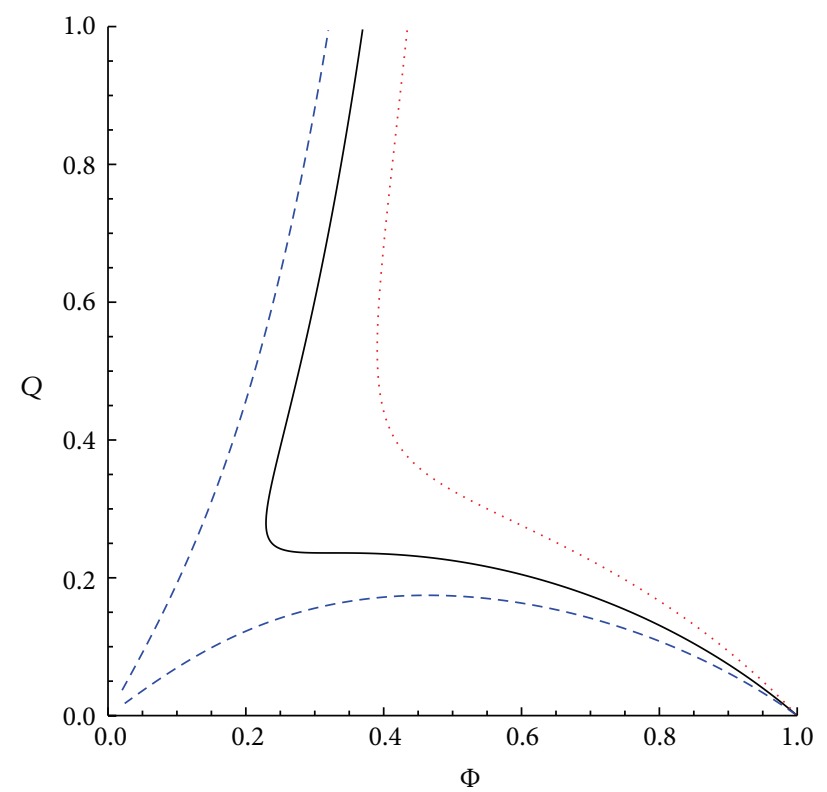

(a) $d=4$

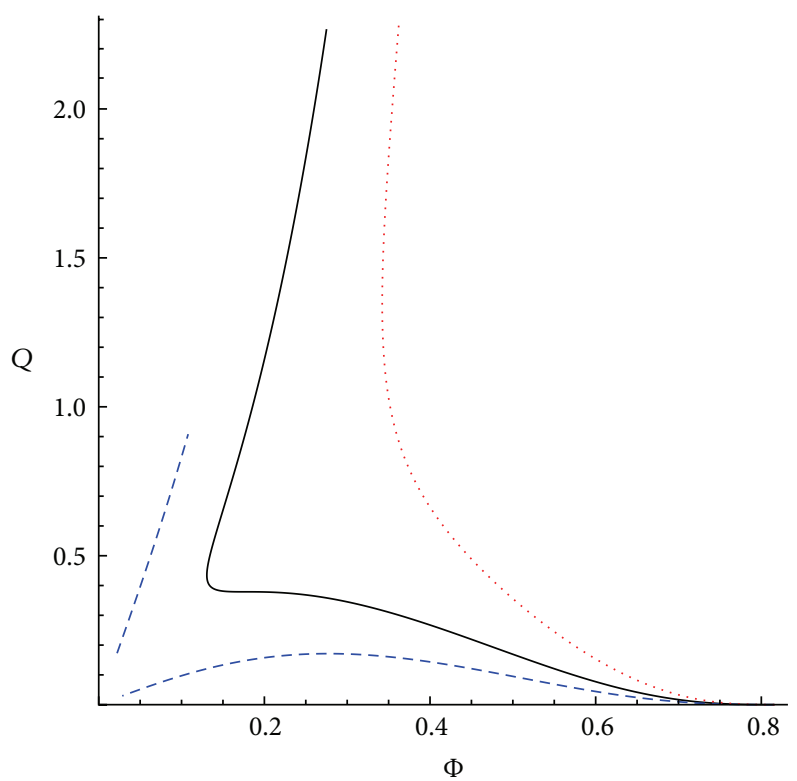

(b) $d=6$

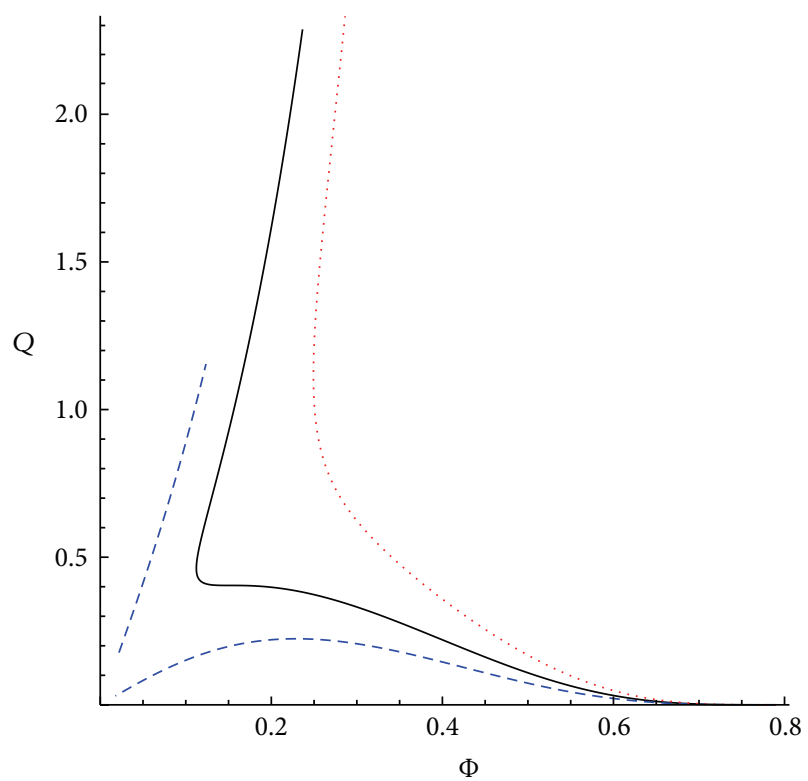

(c) $d=7$

FIgure 4: The $Q-\Phi$ curves for $d=4,6,7$, respectively. From top to bottom the curves correspond to the effective temperatures $0.7 T_{\text {loc }}, T_{\text {loc }}$, and $1.4 T_{\mathrm{loc}}$.

When taking $r_{B}$ and $T_{\text {loc }}$ as constants, we select the dual independent variables $(\sigma, A)$, and the critical position is given by the following conditions:

$$
\begin{aligned}
& \left(\frac{\partial A}{\partial \sigma}\right)_{T_{\mathrm{loc}}, Q}=\frac{1}{8 \pi r_{B}} \\
& \cdot \frac{\left(\partial T_{\mathrm{loc}} / \partial r_{+}\right)_{r_{B}}}{\left(\partial \sigma / \partial r_{B}\right)_{r_{+}}\left(\partial T_{\mathrm{loc}} / \partial r_{+}\right)_{r_{B}}-\left(\partial T_{\mathrm{loc}} / \partial r_{B}\right)_{r_{+}}\left(\partial \sigma / \partial r_{+}\right)_{r_{B}}}
\end{aligned}
$$

$$
\begin{gathered}
=\frac{\left(\partial T_{\mathrm{loc}} / \partial r_{+}\right)_{r_{B}}}{\partial\left(\sigma, T_{\mathrm{loc}}\right) / \partial\left(r_{B}, r_{+}\right)}=f\left(r_{+}, r_{B}\right)=0 \\
\left(\frac{\partial^{2} A}{\partial \sigma^{2}}\right)_{T_{\mathrm{loc}, Q}}=\left(\frac{\partial f\left(r_{+}, r_{B}\right)}{\partial \sigma}\right)_{T_{\mathrm{loc}, Q}}=0
\end{gathered}
$$

Comparing (15), (18), and (19), we can find that the equation of critical point is the same. So we can obtain the same critical point with different independent dual variable in the 


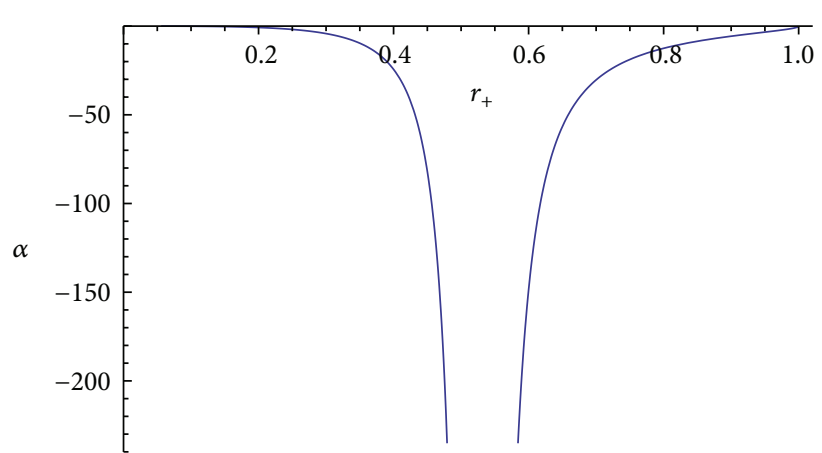

(a) $\alpha-r_{+}$

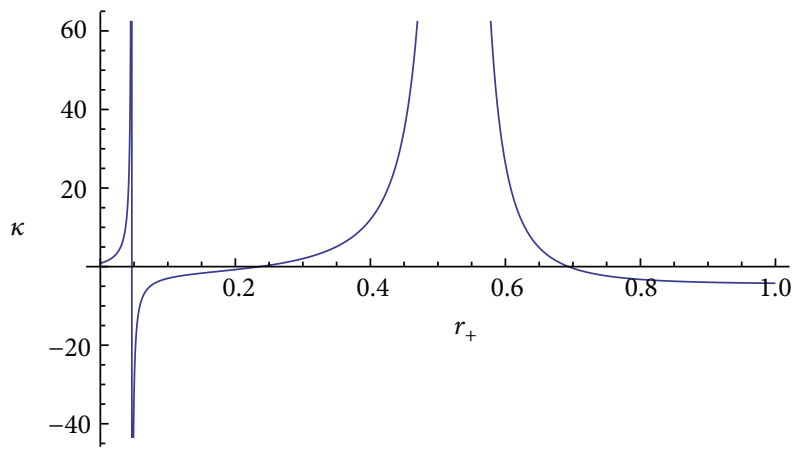

(b) $\kappa-r_{+}$

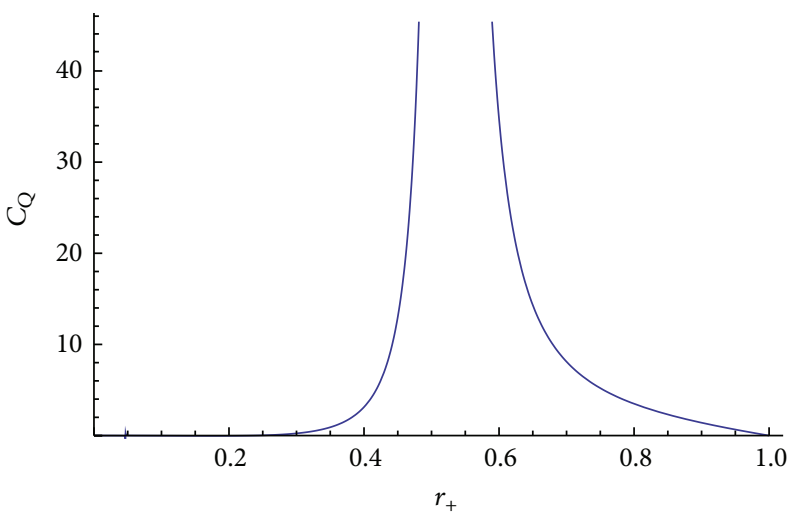

(c) $\mathrm{C}_{\mathrm{Q}^{-}} \mathrm{r}_{+}$

FIGURE 5: $\alpha-r_{+}, \kappa-r_{+}$, and $C_{Q^{-}} r_{+}$curves for higher dimensional charged black hole with $d=4$.

thermodynamic system. The critical point is unaltered with the choice of independent dual variables.

According to Ehrenfest's classification, when the chemical potential and its first derivative are continuous, whereas the second derivative of chemical potential is discontinuous, this kind of phase transition is called the second-order phase transition. For van der Waals system there is no latent heat and the liquid-gas structure does not change suddenly at the critical point. Therefore this kind of phase transition belongs to the second-order phase transition and continuous phase transition. To discuss the critical behaviors of system near the phase transition point with $r_{B}$ unaltered, we will evaluate the second-order partial derivation of chemical potential:

$$
\begin{aligned}
\alpha & =\frac{1}{\Phi}\left(\frac{\partial \Phi}{\partial T_{\mathrm{loc}}}\right)_{\mathrm{Q}}=\frac{1}{\Phi}\left(\frac{\partial \Phi}{\partial r_{+}}\right)_{\mathrm{Q}}\left(\frac{\partial T_{\mathrm{loc}}}{\partial r_{+}}\right)_{\mathrm{Q}}^{-1}, \\
k_{T} & =-\frac{1}{\Phi}\left(\frac{\partial \Phi}{\partial Q}\right)_{T_{\mathrm{loc}}}=-\frac{1}{\Phi}\left[\left(\frac{\partial \Phi}{\partial Q}\right)_{r_{+}}\left(\frac{\partial T_{\mathrm{loc}}}{\partial r_{+}}\right)_{\mathrm{Q}}\right. \\
& \left.-\left(\frac{\partial \Phi}{\partial r_{+}}\right)_{\mathrm{Q}}\left(\frac{\partial T_{\mathrm{loc}}}{\partial Q}\right)_{r_{+}}\right]\left(\frac{\partial T_{\mathrm{loc}}}{\partial r_{+}}\right)_{\mathrm{Q}}^{-1}, \\
C_{\mathrm{Q}} & =T_{\mathrm{loc}}\left(\frac{\partial S}{\partial T_{\mathrm{loc}}}\right)_{\mathrm{Q}}=T_{\mathrm{loc}}\left(\frac{\partial S}{\partial r_{+}}\right)\left(\frac{\partial T_{\mathrm{loc}}}{\partial r_{+}}\right)_{\mathrm{Q}}^{-1} .
\end{aligned}
$$

From (15), (18), and (19), we know that these quantities, $\alpha, k_{T}$, and $C_{Q}$, obtained in (20), approach infinity at the critical point. So the phase transition, which happens in the black hole thermodynamic ensemble, is a continuous phase transition. The Gibbs free energy and the first-order partial derivation for these two phases are continuous at the critical point of continuous phase. We also depict the curves of $\alpha$ $r_{+}, \mathcal{k}-r_{+}$, and $C_{Q^{-} r_{+}}$in Figure 5, respectively, at the constant pressure. From these curves, we find that the specific heat of black hole thermodynamic system at constant charge $C_{\mathrm{Q}}$, the expansion coefficient $\alpha$, and the compressibility $\kappa$ have infinite peak.

For usual thermodynamic system, the entropy change and volume change are equivalent, respectively, as $d v^{1}=d v^{2}$ and $d S^{1}=d S^{2}$, near critical point; Ehrenfest had calculated the Ehrenfest equation:

$$
\begin{aligned}
& \frac{d P}{d T}=\frac{\alpha^{1}-\alpha^{2}}{k_{T}^{1}-k_{T}^{2}}, \\
& \frac{d P}{d T}=\frac{C_{P}^{1}-C_{P}^{2}}{T v\left(\alpha^{1}-\alpha^{2}\right)},
\end{aligned}
$$

in which superscripts 1 and 2 represent phases 1 and 2, respectively. Recently, the Ehrenfest equation for AdS black 
hole thermodynamic system [29-35, 41] has been studied and the Prigogine-Defay (PD) relation is obtained:

$$
\Pi=\frac{\Delta C_{P} \Delta k_{T}}{T V(\Delta \alpha)^{2}}=1
$$

For the black hole thermodynamic system, we can rewrite the equation of Ehrenfest as

$$
\begin{aligned}
& \left(\frac{\partial Q}{\partial T_{\mathrm{loc}}}\right)_{\Phi}=\frac{\alpha_{2}-\alpha_{1}}{k_{T_{2}}-k_{T_{1}}}=\frac{\Delta \alpha}{\Delta k_{T}} \\
& \left(\frac{\partial Q}{\partial T_{\mathrm{loc}}}\right)_{S}=\frac{C_{\mathrm{Q}_{2}}-C_{\mathrm{Q}_{1}}}{T_{\mathrm{loc}} \Phi\left(\alpha_{2}-\alpha_{1}\right)}=\frac{\Delta C_{\mathrm{Q}}}{T_{\mathrm{loc}} \Phi \Delta \alpha} .
\end{aligned}
$$

Using

$$
\begin{aligned}
\left(\frac{\partial T_{\mathrm{loc}}}{\partial Q}\right)_{\Phi}= & \left(\frac{\partial T_{\mathrm{loc}}}{\partial Q}\right)_{S} \\
& -\left(\frac{\partial T_{\mathrm{loc}}}{\partial S}\right)_{Q}\left(\frac{\partial \Phi}{\partial Q}\right)_{S}\left(\frac{\partial \Phi}{\partial S}\right)_{Q}^{-1},
\end{aligned}
$$

according to (18), the critical points satisfy

$$
\left(\frac{\partial T_{\mathrm{loc}}}{\partial r_{+}}\right)_{\mathrm{Q}}=\left(\frac{\partial T_{\mathrm{loc}}}{\partial S}\right)_{\mathrm{Q}}\left(\frac{\partial S}{\partial r_{+}}\right)_{\mathrm{Q}}=0 \text {. }
$$

From (5), we know

$$
\left(\frac{\partial S}{\partial r_{+}}\right)_{Q}=\frac{\omega_{d-2}(d-2) r_{+}^{d-3}}{4} \neq 0
$$

So, (24) can be written as

$$
\left(\frac{\partial T_{\mathrm{loc}}}{\partial Q}\right)_{\Phi}^{c}=\left(\frac{\partial T_{\mathrm{loc}}}{\partial Q}\right)_{S}^{c}
$$

Substituting (27) into (23), the Prigogine-Defay (PD) ratio (П) can be calculated as

$$
\Pi=\frac{\Delta C_{\mathrm{Q}} \Delta \kappa_{T}}{T_{\mathrm{loc}} \Phi(\Delta \alpha)^{2}}=1 .
$$

Hence, when $Q$ is constant, the phase transition occurring at $T_{\text {loc }}=T_{\text {loc }}^{c}$ is a second-order equilibrium transition. This is true in spite of the fact that the phase transition curves are smeared and divergent near the critical point. This result is in agreement with the result of the AdS black holes.

\section{Discussion and Conclusions}

In this work, to study the properties of black hole in asymptotically flat spacetime, we built a stable black hole thermodynamic ensemble in the view of thermodynamics. we calculated the local energy and local temperature and find that these state parameters satisfy the first law of thermodynamic equation (12). Based on this condition, we analyze the critical behavior of black hole thermodynamic ensemble through taking the state parameters $(Q, \Phi)$ of black hole thermodynamic ensemble corresponding to the state parameters $(P, V)$ of van der Waals system, respectively. We obtain the critical point of black hole thermodynamic system and find that the critical point is independent of the dual independent variables we selected. This result for asymptotically flat space is consistent with the conclusion for AdS spacetime [1] and is an intrinsic property of black hole thermodynamic ensemble. Firstly, from Table 1 we can find that the critical temperature and critical charge of black hole ensemble increase with the spacetime dimensional increase, which is similar to the critical behavior of AdS black hole. Secondly, the second partial derivatives $\alpha, \kappa_{T}$, and $C_{Q}$ of Gibbs free energy of black hole thermodynamic ensemble are divergent at the critical point; the result is the same as the result of AdS spacetime. Finally, the Prigogine-Defay (PD) relation at critical point in black hole thermodynamic ensemble is consistent with the AdS black hole. The PD ratio satisfies (28) and is independent of spacetime dimension.

In the black hole thermodynamic ensemble, we built a concentric spherical cavity, whose radii are fixed and larger than the ones of black hole, to achieve the condition of thermodynamic stability for black hole ensemble. The reason, for which AdS black hole can achieve the thermodynamic stability, is that there is a cosmology constant $\Lambda$. Through comparing the critical behavior of thermodynamic ensemble with the ones of AdS spacetime, we can argue that the radii $r_{B}$ of concentric spherical cavity are related to the cosmology constant $\Lambda$ in the thermodynamic view.

\section{Competing Interests}

The authors declare that they have no competing interests.

\section{Acknowledgments}

The authors would like to thank Dr. Meng-Sen Ma and HuiHua Zhao for their indispensable discussions and comments. Huaifan Li would like to acknowledge the Program for the Innovative Talents of Higher Learning Institutions of Shanxi and is also grateful to KITPC for its hospitality and its partial support during the completion of this work. This work was supported in part by the National Natural Science Foundation of China (Grant nos. 11075098, 11175109, and 11475108), the Young Scientists Fund of the National Natural Science Foundation of China (Grant no. 11205097), the Natural Science Foundation for Young Scientists of Shanxi Province, China (Grant no. 2012021003-4), and the Natural Science Foundation of Datong city (Grant no. 20150110).

\section{References}

[1] D. Kubiznak and R. B. Mann, " $P-V$ criticality of charged AdS black holes," Journal of High Energy Physics, vol. 2012, article 33, 2012.

[2] B. P. Dolan, D. Kastor, D. Kubizňák, R. B. Mann, and J. Traschen, "Thermodynamic volumes and isoperimetric inequalities for de Sitter black holes," Physical Review D, vol. 87, no. 10, Article ID 104017, 2013. 
[3] S. Gunasekaran, D. Kubiznak, and R. B. Mann, "Extended phase space thermodynamics for charged and rotating black holes and Born-Infeld vacuum polarization," Journal of High Energy Physics, vol. 2012, article 110, 2012.

[4] M. Cvetic, G. W. Gibbons, D. Kubiznak, and C. N. Pope, "Black hole enthalpy and an entropy inequality for the thermodynamic volume," Physical Review D, vol. 84, no. 2, Article ID 024037, 2011.

[5] R.-G. Cai, L.-M. Cao, L. Li, and R.-Q. Yang, "P-V criticality in the extended phase space of Gauss-Bonnet black holes in AdS space," Journal of High Energy Physics, vol. 2013, no. 9, article 005, 2013.

[6] M. S. Ma, F. Liu, and R. Zhao, "Continuous phase transition and critical behaviors of 3D black hole with torsion," Classical and Quantum Gravity, vol. 31, no. 9, Article ID 095001, 2014.

[7] S. H. Hendi and B. E. Panah, "Thermodynamics of rotating black branes in Gauss-Bonnet-nonlinear Maxwell gravity," Physics Letters B, vol. 684, no. 2-3, pp. 77-84, 2010.

[8] M.-S. Ma, "Thermodynamics and phase transition of black hole in an asymptotically safe gravity," Physics Letters B, vol. 735, pp. 45-50, 2014.

[9] S.-W. Wei and Y.-X. Liu, "Triple points and phase diagrams in the extended phase space of charged Gauss-Bonnet black holes in AdS space," Physical Review D, vol. 90, no. 4, Article ID 044057, 2014.

[10] S. W. Wei and Y. X. Liu, "Insight into the microscopic structure of an AdS black hole from a thermodynamical phase transition," Physical Review Letters, vol. 115, no. 11, Article ID 111302, 2015.

[11] Y. Q. Liu, D.-C. Zou, and B. Wang, "Signature of the Van der Waals like small-large charged AdS black hole phase transition in quasinormal modes," Journal of High Energy Physics, vol. 1409, p. 179, 2014.

[12] S. H. Hendi, S. Panahiyan, and B. Eslam Panah, "Extended phase space of Black Holes in Lovelock gravity with nonlinear electrodynamics," Progress of Theoretical and Experimental Physics, no. 10, Article ID 103E01, 2015.

[13] S. H. Hendi, R. Moradi, and Z. Armanfard, "Extended phase space thermodynamics and P-V criticality: BransDicke-Born-Infeld vs Einstein-BI-dilaton black holes," https://arxiv.org/abs/1511.02761.

[14] R. Zhao, H. H. Zhao, M. S. Ma, and L. C. Zhang, "On the critical phenomena and thermodynamics of charged topological dilaton AdS black holes," European Physical Journal C, vol. 73, article 2645, 2013.

[15] M. Urano and A. Tomimatsu, "The mechanical first law of black hole spacetimes with a cosmological constant and its application to the Schwarzschild-de Sitter spacetime," Classical and Quantum Gravity, vol. 26, no. 10, 2009.

[16] M. S. Ma, H. H. Zhao, L. C. Zhang, and R. Zhao, "Existence condition and phase transition of Reissner-Nordström-de Sitter black hole," International Journal of Modern Physics A, vol. 29, no. 9, Article ID 1450050, 12 pages, 2014.

[17] H. H. Zhao, L. C. Zhang, M. S. Ma, and R. Zhao, " $P-V$ criticality of higher dimensional charged topological dilaton de Sitter black holes," Physical Review D, vol. 90, no. 6, Article ID 064018, 2014.

[18] M.-S. Ma, L.-C. Zhang, H.-H. Zhao, and R. Zhao, "Phase transition of the higher dimensional charged Gauss-Bonnet black hole in de Sitter spacetime," Advances in High Energy Physics, vol. 2015, Article ID 134815, 8 pages, 2015.
[19] X. Y. Guo, H. F. Li, L. C. Zhang, and R. Zhao, "Thermodynamics and phase transition in the Kerr-de Sitter black hole," Physical Review D, vol. 91, no. 8, Article ID 084009, 2015.

[20] R. Zhao, M. Ma, H. Zhao, and L. Zhang, “The critical phenomena and thermodynamics of the Reissner-Nordstrom-de Sitter black hole," Advances in High Energy Physics, vol. 2014, Article ID 124854, 6 pages, 2014.

[21] J. W. York, "Black-hole thermodynamics and the Euclidean Einstein action," Physical Review D, vol. 33, no. 8, pp. 20922099, 1986.

[22] H. W. Braden, J. D. Brown, B. F. Whiting, and J. York, "Charged black hole in a grand canonical ensemble," Physical Review D, vol. 42, no. 10, pp. 3376-3385, 1990.

[23] A. P. Lundgren, "Charged black hole in a canonical ensemble," Physical Review D, vol. 77, no. 4, Article ID 044014, 8 pages, 2008.

[24] S. Carlip and S. Vaidya, "Phys transitions and critical behavior for charged black holes," Classical and Quantum Gravity, vol. 20, no. 16, pp. 3827-3837, 2003.

[25] F. Capela and G. Nardini, "Hairy black holes in massive gravity: thermodynamics and phase structure," Physical Review D, vol. 86, no. 2, Article ID 024030, 12 pages, 2012.

[26] J. X. Lu, S. Roy, and Z. Xiao, "Phase transitions and critical behavior of black branes in canonical ensemble," Journal of High Energy Physics, vol. 2011, no. 1, article 133, 2011.

[27] J. X. Lu, S. Roy, and Z. Xiao, “The enriched phase structure of black branes in canonical ensemble," Nuclear Physics B, vol. 854, no. 3, pp. 913-925, 2012.

[28] M. Eune, Y. Gim, and W. Kim, “Thermodynamic phase transition based on the nonsingular temperature," Physical Review D, vol. 91, no. 4, Article ID 044037, 7 pages, 2015.

[29] J.-X. Mo and W.-B. Liu, "Ehrenfest scheme for P-V criticality in the extended phase space of black holes," Physics Letters B, vol. 727, no. 1-3, pp. 336-339, 2013.

[30] J. X. Mo and W. B. Liu, " $P-V$ criticality of topological black holes in Lovelock-Born Infeld gravity," The European Physical Journal C, vol. 74, article 2836, 2014.

[31] J. X. Mo, G. Q. Li, and W. B. Liu, "Another novel Ehrenfest scheme for $P-V$ criticality of RN-AdS black holes," Physics Letters B, vol. 730, pp. 111-114, 2014.

[32] J. X. Mo, X. X. Zeng, G. Q. Li, X. Jiang, and W. B. Liu, "A unified phase transition picture of the charged topological black hole in Hořava-Lifshitz gravity," Journal of High Energy Physics, vol. 2013, 56 pages, 2013.

[33] J.-X. Mo, "Ehrenfest scheme for the extended phase space of $f(R)$ black holes," Europhysics Letters, vol. 105, no. 2, Article ID 20003, 2014.

[34] L.-C. Zhang and R. Zhao, “The universal Ehrenfest scheme on black holes," Modern Physics Letters A, vol. 30, no. 36, Article ID 1550187, 6 pages, 2015.

[35] Z. Zhao and J. Jing, "Ehrenfest scheme for complex thermodynamic systems in full phase space," Journal of High Energy Physics, vol. 2014, no. 11, article 37, 2014.

[36] J. Suresh, R. Tharanath, N. Varghese, and V. C. Kuriakose, “The thermodynamics and thermodynamic geometry of the Park black hole," The European Physical Journal C, vol. 74, article 2819, 2014.

[37] S. Ali, H. Mansoori, and B. Mirza, "Correspondence of phase transition points and singularities of thermodynamic geometry of black holes," The European Physical Journal C, vol. 74, article 2681, 2014. 
[38] R. Tharanath, J. Suresh, and V. C. Kuriakose, "Phase transitions and geometrothermodynamics of regular black holes," General Relativity and Gravitation, vol. 47, article 46, 2015.

[39] J.-X. Zhao, M.-S. Ma, L.-C. Zhang, H.-H. Zhao, and R. Zhao, "The equal area law of asymptotically AdS black holes in extended phase space," Astrophysics and Space Science, vol. 352, no. 2, pp. 763-768, 2014.

[40] S.-Q. Lan, J.-X. Mo, and W.-B. Liu, "A note on Maxwell's equal area law for black hole phase transition," The European Physical Journal C, vol. 75, no. 9, article 419, 2015.

[41] R. Banerjee and D. Roychowdhury, "Thermodynamics of phase transition in higher dimensional AdS black holes," Journal of High Energy Physics, vol. 2011, no. 11, article 4, 2011. 

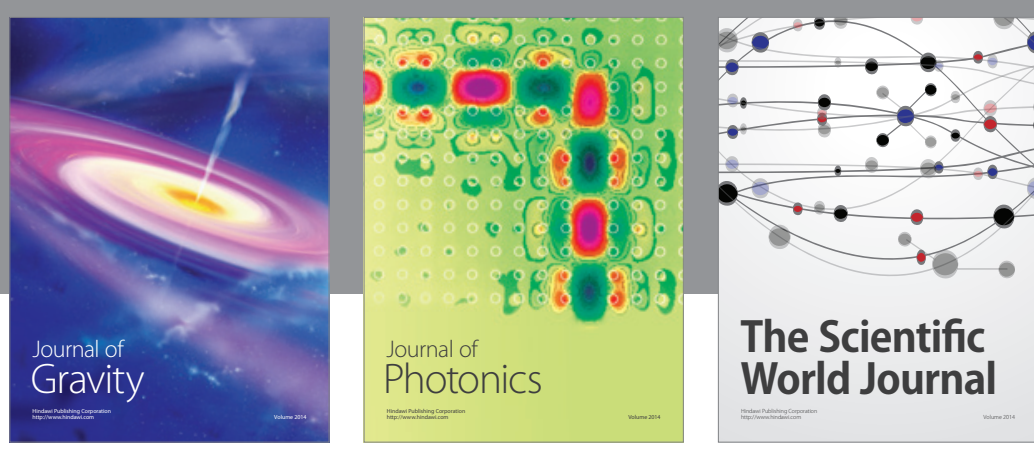

The Scientific World Journal
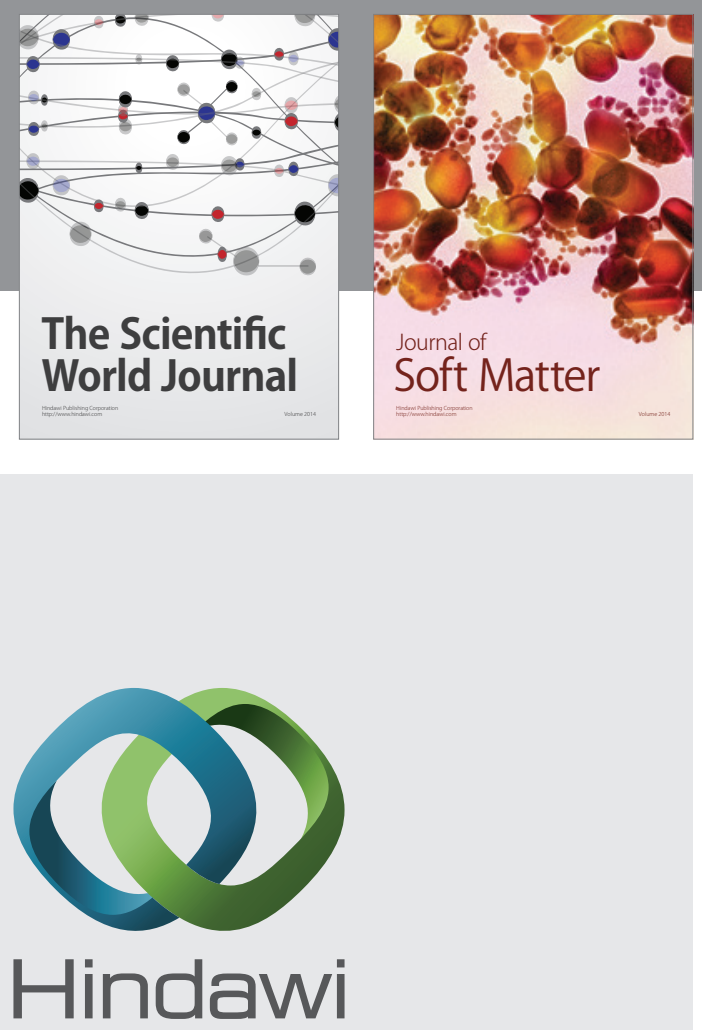

Submit your manuscripts at

http://www.hindawi.com

nternational Journal of

Statistical Mechanics
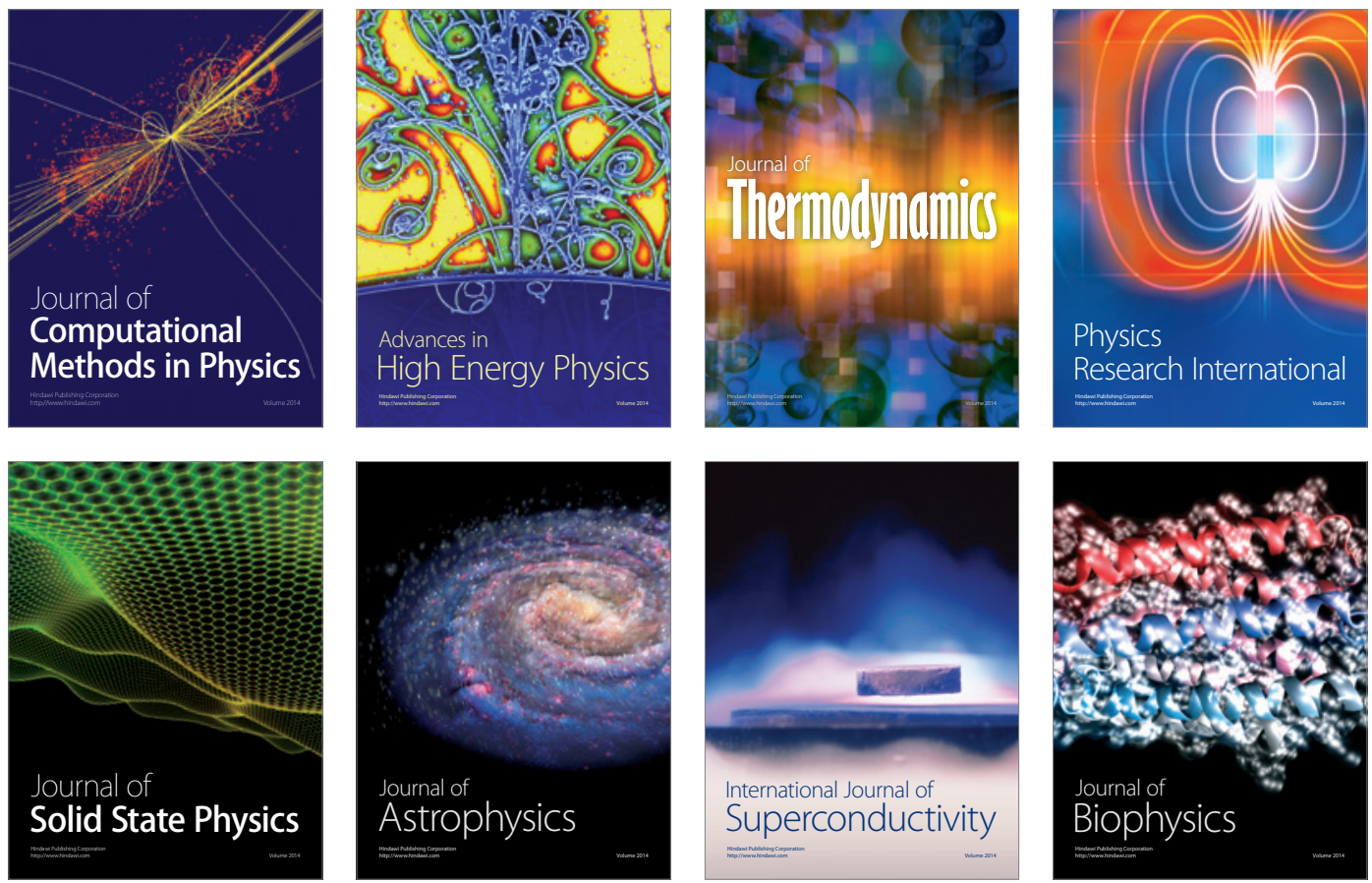
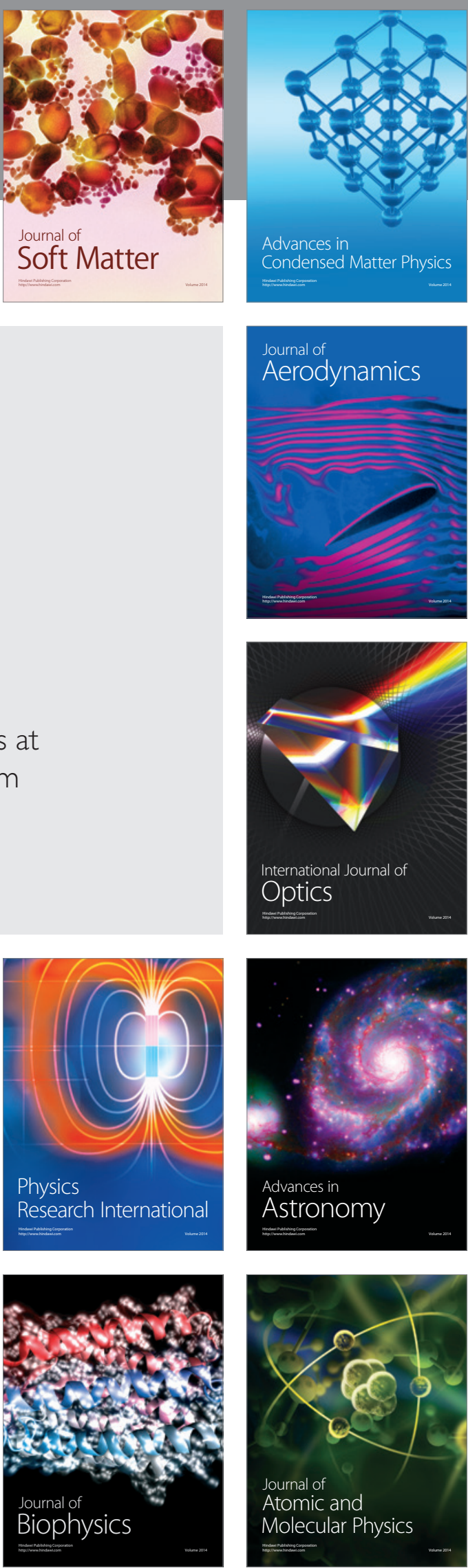